\title{
VARIAÇÃO SAZONAL DE LEPIDÓPTEROS MINADORES E SEUS PARASITOIDES EM PLANTAS DE CRESCIMENTO ESPONTÂNEO EM POMAR ORGÂNICO DE CITROS EM MONTENEGRO, RS, BRASIL
}

\author{
J.P. dos Santos", L.R. Redaelli², F.K. Dal Soglio², E. Foelkel², V.A. Costa ${ }^{3}$ \\ ${ }^{1}$ EPAGRI, Estação Experimental de Caçador, Laboratório de Entomologia, CP 591, CEP 89500-000, Caçador, \\ SC, Brasil. E-mail: janapereira@epagri.sc.gov.br
}

\section{RESUMO}

Phyllocnistis citrella (Lepidoptera: Gracillariidae), conhecido popularmente como "minador-dasfolhas-dos-citros", é um dos principais insetos-praga da citricultura em vários países. Este trabalho teve como objetivos determinar as espécies e a variação sazonal de lepidópteros minadores e seus parasitoides, presentes na vegetação de crescimento espontâneo de um pomar orgânico de citros, a fim de verificar se $P$. citrella apresenta outras plantas como hospedeiras e se os parasitoides de outros minadores são os mesmos relatados para esta praga. $\mathrm{O}$ trabalho foi conduzido em Montenegro, RS, em um pomar do tangoreiro 'Murcott'. Realizaram-se amostragens quinzenais, de maio/ 2003 a maio/2004, coletando-se em cada ocasião todas as folhas com minas, na área delimitada por um aro de $0,28 \mathrm{~m}^{2}$, que era lançado nas linhas e nas entrelinhas de 30 árvores sorteadas. No laboratório registrou-se o número de larvas e pupas de minadores por folha, mantidas até a emergência dos lepidópteros ou parasitoides. Registrou-se 11 espécies de lepidópteros minadores pertencentes às famílias Gracillariidae, Gelechiidae, Acrolepiidae, Nepticulidae e Tischeriidae; 15 espécies de plantas hospedeiras distribuídas em nove famílias e 12 espécies de himenópteros, das famílias Eulophidae e Braconidae. O número de larvas e pupas de minadores correlacionou-se negativamente com a temperatura média e a umidade relativa média. $P$. citrella não foi observada nas plantas espontâneas. Espécies de parasitoides incluídas nos gêneros Chrysocharis, Closterocerus, Sympiesis e Bracon já foram relatadas parasitando $P$. citrella em várias regiões do mundo e podem ser possíveis agentes de controle biológico deste inseto na área de estudo.

PALAVRAS-CHAVE: Insecta, Hymenoptera, biodiversidade, inimigos naturais, dinâmica popaqlacional.

\section{ABSTRACT}

SEASONAL VARIATION OF LEPIDOPTERA LEAFMINERS AND their PARASITOIDS IN SPONTANEOUS VEGETATION IN AN ORGANIC CITRUS ORCHARD IN MONTENEGRO, RS, BRAZIL. Phyllocnistis citrella (Lepidoptera: Gracillariidae), known popularly as "citrus leafminer," is one of the main insect-pests of citrus orchards in many countries. This study was aimed to determine the species and the seasonal variation of Lepidoptera leafminers and their parasitoids, present in the vegetation spontaneously growing in a citrus orchard, to verify if $P$. citrella is present in other host plants and if the parasitoids of other leafminers are the same ones reported for this insect-pest. The work was conducted in Montenegro, state of Rio Grande do Sul, Brazil, in an organic orchard of the Murcott cultivar. Samplings were taken fortnightly, from May 2003 to May 2004, collecting on each occasion all the plants with mines found in an area delimited by a $0.28 \mathrm{~m}^{2}$ ring thrown in the lines and between the lines of 30 randomly chosen trees. In the lab, the number of larvae and pupae per leaf of leafminers were recorded. The leaves were maintained until emergence of leafminers or parasitoids. Counting revealed 11 species of Lepidoptera leafminers of the families Gracillariidae, Acrolepiidae, Nepticulidae and Tischeriidae; 15 species of leafminer host plants of 9 families, and 12 species of hymenopteran parasitoids of the families Eulophidae and Braconidae. The leafminers larvae and pupae presented a negative association with temperature and air relative humidity. $P$. citrella was not observed in the spontaneous plants. Parasitoid species included in the genera Chrysocharis, Closterocerus, Sympiesis and Bracon have been previous reported as parasitizing P. citrella in several countries, and could be possible agents of biological control of this insect in the study area.

KEY WORDS: Insecta, Hymenoptera, biodiversity, natural enemies, population dynamics.

${ }^{2}$ Universidade Federal do Rio Grande do Sul, Departamento de Fitossanidade, Porto Alegre, RS, Brasil.

${ }^{3}$ Instituto Biológico, Centro Experimental Central, Campinas, SP, Brasil. 


\section{INTRODUÇÃO}

O conhecimento da composição, estrutura e funcionamento da comunidade de insetos presentes em plantas de crescimento espontâneo fornece subsídios para o manejo dos agroecossistemas. A compreensão dos processos responsáveis pelas variações que ocorrem nessa comunidade permite a realização de previsões a respeito das flutuações populacionais, que pode ser utilizada no manejo de populações, para a conservação ou para o controle de populações eruptivas (BEGON; MORTIMER, 1986; BEGON et al., 1990).

Insetos das ordens Coleoptera, Diptera, Hymenoptera e Lepidoptera evoluíram o hábito minador. Os representantes dessas ordens apresentam metamorfose completa e um grau mais alto de especialização em relação aos insetos de outras ordens (Hespenheide, 1991; Verdú, 1996; BYers, 2002; CARLEtTI, 2004). A ordem que apresenta maior diversidade de espécies minadoras é Lepidoptera, seguida por Diptera, Coleoptera e Hymenoptera, porém, essa diversidade pode mudar de acordo com o local (Hespenheide, 1991; Verdú, 1996).

Os minadores frequentemente relatados em Lepidoptera são representantes das famílias Gracillariidae, Gelechiidae, Nepticulidae, Tischeriidae (Hespenheide, 1991; BYers, 2002), Coleophoridae, Eriocraniidae, Glyphipterygidae, Incurvariidae, Lyonetiidae, Lithocolletidae, Micropterygidae, Noctuidae, Pyralidae e Tortricidae (CARLETTI, 2004).

Um importante inseto-praga com hábito minador é Phyllocnistis citrella Stainton, 1856 (Lepidoptera, Gracillariidae), conhecido popularmente como "minador-das-folhas-dos-citros" (HEPPNER, 1993). Este microlepidóptero, nativo da Ásia, ataca folhas novas das brotações de plantas de citros, fazendo galerias em forma de serpentina, provocando atrofia das folhas que ficam de coloração prateada e redução da área fotossintética (SCHAFFER et al., 1997). Plantas hospedeiras alternativas, principalmente da família Rutaceae, também podem servir para o desenvolvimento das larvas desse minador (HEPPNER, 1993; KNAPP et al., 1995). Em relação a inimigos naturais, no Brasil são conhecidas muitas espécies nativas de parasitoides que se adaptaram e utilizaram $P$. citrella como hospedeiro (JAHNKE et al., 2005). Sendo que os minadores presentes em plantas de crescimento espontâneo que ocorrem em pomares podem servir dereservatório de inimigos naturais para essa praga.

Osinsetos minadores apresentam um hábitatmuito exposto e relativamente fácil de ser localizado por inimigos naturais, sendo atacados por uma grande variedade de espécies (VERDú, 1996), principalmente parasitoides.

Há uma lacuna no conhecimento, sobretudo no Brasil, a respeito de insetos minadores, seus hospedeiros e inimigos naturais. Assim, o presente trabalho teve como objetivos determinar as espéciese a variação sazonal de lepidópteros minadores e seus parasitoides presentes na vegetação de crescimento espontâneo, em pomar orgânico de citros, a fim de verificar se $P$. citrella apresenta outras plantas como hospedeiras e se os parasitoides de outros mimadores são os mesmos relatados para $P$. citrella.

\section{MATERIAL E MÉTODOS}

O trabalho foi desenvolvido em um pomar orgânico de tangoreiro Murcott (Citrus sinensis L. Osbeck $x$ Citrus reticulata Blanco), enxertado sobre Poncirus trifoliata (L.) Raf, no Município de Montenegro (29 $68^{\prime}$ S e $\left.51^{\circ} 46^{\prime} \mathrm{W}\right)$, localizado no vale do Rio Caí, RS. A área tem 0,6 ha, com 370 plantas com 12 anos de idade, distribuídas no espaçamento entre plantas de $3,5 \mathrm{~m}$ e entrelinhas de $5 \mathrm{~m}$.

A temperatura média anual da região é de $19,4^{\circ}$ C, apresentando chuvas abundantes $(1.537 \mathrm{~mm} / \mathrm{ano})$ e bem distribuídas (Rodriguez et al., 1991).

Quinzenalmente, de maio de 2003 a maio de 2004, foram realizadas amostragens na vegetação que cresce espontaneamente entre as plantas de citros e nas entrelinhas. Para a coleta das amostras foram sorteadas 30 árvores por ocasião de amostragem, nas quais se coletou por ponto, duas amostras, um ponto representou a entrelinha e o outro a linha. Para o sorteio dos pontos, as plantas de citros foram numeradas e, através do programa BioEstat ${ }^{\circledR}$ (AYres et al., 2000) em cada ocasião sortearam-se números que corresponderam aos pontos amostrais. Retirou-se em cada ocasião 60 amostras que consistiram de todas as plantas com minas presentes num círculo de $0,28 \mathrm{~m}^{2}$, delimitado por um aro de pvc com $60 \mathrm{~cm}$ de diâmetro, adaptação feita do método do quadrilátero, proposto por SouTHWOOD (1978), e que era lançado nas linhas e entrelinhas de 30 árvores sorteadas.

As plantas foram colocadas individualmente em sacos plásticos e levadas até o laboratório para triagem. No Laboratório de Biologia, Ecologia e Controle Biológico de Insetos, do Departamento de Fitossanidade da Faculdade de Agronomia, da Universidade Federal do Rio Grande do Sul (UFRGS), as folhas foram examinadas com o auxílio de microscópio estereoscópio, registrando-se o número de larvas e/ou pupas de minadores. As folhas minadas foram acondicionadas em placas de Petri de $9 \mathrm{~cm}$ de diâmetro e $1,5 \mathrm{~cm}$ de altura ou em caixas gerbox de 11,2 cm de diâmetro e 3,4 cm de altura, que foram mantidas em câmara climatizada (fotofase de 12 horas, $25^{\circ} \mathrm{C} \pm 1^{\circ} \mathrm{C}$ ) até a emergência dos adultos de minadores e/ou de parasitoides. Para favorecer o desenvolvimento completo dos insetos, mantiveram-se as folhas túrgidas colocando-se no 
pecíolo destas, um chumaço de algodão que era umedecido diariamente.

Os parasitoides emergidos foram conservados individualmente em recipientes tipo "eppendorf" contendo álcool $70 \%$ e os lepidópteros foram conservados em envelopes entomológicos. A identificação das famílias de parasitoides foi efetuada com o auxílio das chaves dicotômicas de GAULD; HANSON (1995). Paraidentificação a nível genérico eespecífico, tanto os parasitoides quanto os lepidópteros foram enviados a especialistas.

Foram feitas exsicatas das plantas hospedeiras doslepidópteros minadores, que foramidentificadas com o auxílio da bibliografia e por comparação com o acervo de plantas do herbário do Departamento de Botânica da UFRGS.

A influência dos fatores meteorológicos sobre as larvas e pupas de minadores foi avaliada através da correlação de Pearson, o nível de significância adotado foi de $5 \%$. Os registros diários dos dados meteorológicos referentes à temperatura mínima, média e máxima, precipitação pluviométrica e umidade relativa do ar foram fornecidos pela Estação Experimental da FEPAGRO (Fundação Estadual de Pesquisa Agropecuária) situada em Taquari, RS, município próximo a Montenegro. Nas análises utilizou-se para cada ocasião de amostragem, os valores correspondentes à média dos registros diários da quinzena anterior a cada amostragem.

\section{RESULTADOS}

Em 27 amostragens foram coletadas 1.030 larvas e151 pupas de lepidópteros minadores, registrando- -se a emergência de 332 indivíduos, distribuídos em 11 espécies, pertencentes às famílias Gracillariidae, Gelechiidae, Acrolepiidae, Nepticulidae, Tischeriidae e em uma não identificada. Foram coletados 202 himenópteros parasitoides, pertencentes a 12 espécies, distribuídas nas famílias Eulophidae e Braconidae (Tabela 1).

Onúmero de espécies e de famílias de minadores foi crescente ao longo das amostragens, entretanto, nas últimas coletas verifica-se a tendência de estabilização (Fig. 1A e 1B).

Registraram-se em Gracillariidae as espécies Cameraria sp.; Phyllocnistis sp.; Porphyrosela sp., e uma espécie identificada apenas até subfamília (Lithocolletinae), por se tratar de um gênero novo. Em Gelechiidae foram obtidas três espécies cuja identificação específica não foi possível até o presente, por se tratar de possíveis gêneros novos (DAvis, comunicação pessoal).

Foi registrada uma espécie em cada uma das seguintes famílias: Acrolepiidae (Antispastis xylophragma Meyrick, 1926); Nepticulidae (Stigmella sp.) e em Tischeriidae (Tischeria sp.) (Tabela 1).

Foram obtidos 5 indivíduos não identificados ao nível de família, registrados neste estudo como "morfoespécie não identificada". De acordo com DAVIS (comunicação pessoal), provavelmente trata-se de uma família nova.

Com relação aos parasitoides, em Eulophidae foram constatadas três espécies do gênero Chrysocharis, uma de Sympiesis, além de Closterocerus coffeellae Ihering, 1913 (Tabela 1). Na família Braconidaeforam registradas duas espécies de Bracon e de Pholetesor; e apenas uma espécie de Dolichogenidea, Orgilus e Stiropius.
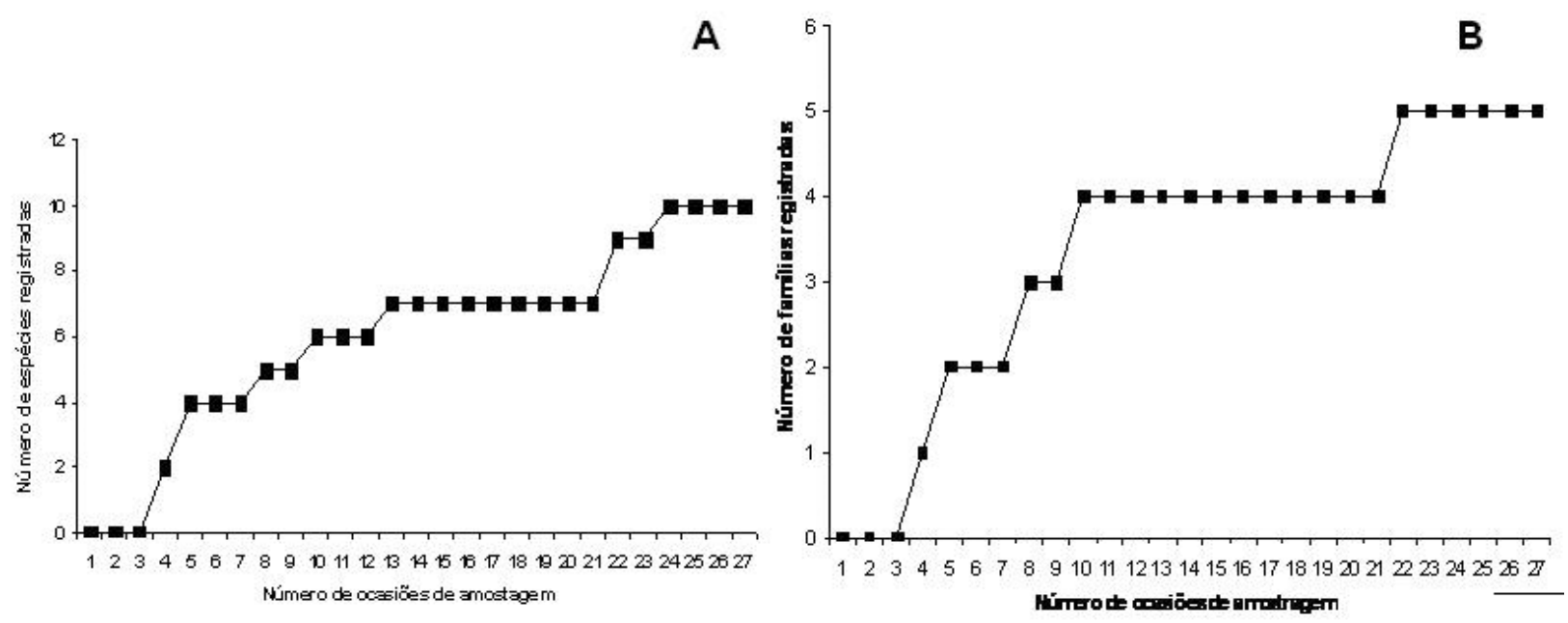

Fig. 1- Número cumulativo de (A) espécies e (B) famílias de lepidópteros minadores, obtidas em sucessivas amostragens em plantas de crescimento espontâneo, em pomar de tangor 'Murcott'., no Município de Montenegro, RS (maio de 2003 a maio de 2004). 
Tabela 1 - Plantas hospedeiras, lepidópteros minadores e seus respectivos parasitoides, presentes em pomar de 'Murcott'. Montenegro, RS (maio de 2003 a maio de 2004).

\begin{tabular}{|c|c|c|}
\hline $\begin{array}{l}\text { Plantas hospedeiras } \\
\text { família-espécie } \\
\text { (Nome comum) }\end{array}$ & $\begin{array}{l}\text { Lepidópteros minadores } \\
\text { família-espécie } \\
\text { (No de emergidos) }\end{array}$ & $\begin{array}{l}\text { Parasitoides família-espécie } \\
\text { (Nº de emergidos) }\end{array}$ \\
\hline Asteraceae & Gracilariidae & \\
\hline \multirow[t]{8}{*}{ Baccharis anômala (cambará-de-cipó) } & Phyllocnistis sp..$^{(1)}(23)$ & $\begin{array}{l}\text { Eulophidae- Closterocerus. coffeellae (139); } \\
\text { Chrysocharis sp. } 1^{(2)}(3) ; \text { Chrysocharis sp. } 2^{(2)} \\
\text { (1); Chrysocharis sp. } 3 \text { (1) } \\
\text { Braconidae- Pholetesor sp. } 2 \text { (1); } \\
\text { Dolichogenidea sp. (1); Orgilus sp. (1) }\end{array}$ \\
\hline & Lithocolletinae gen. nov. ${ }^{(3)}(164)$ & $\begin{array}{l}\text { Eulophidae- C. coffeellae (6); Chrysocharis sp. } \\
2 \text { (1) } \\
\text { Braconidae-Pholetesorsp. 2(3); Stiropius sp.(2) }\end{array}$ \\
\hline & Cameraria sp. (1) & -------------- \\
\hline & $\begin{array}{l}\text { Acrolepiidae } \\
\text { Antispastis xylophragma (5) }\end{array}$ & $\begin{array}{l}\text { Eulophidae- C. coffeellae (2) } \\
\text { Braconidae- Pholetesor sp. } 1 \text { (1) }\end{array}$ \\
\hline & Gelechiidae & \\
\hline & Morfoespécie $1^{(4)}(1)$ & Eulophidae- Sympiesis sp. (2) \\
\hline & Morfoespécie não identificada(5) $(3)$ & ----------- \\
\hline & $\begin{array}{l}\text { Nepiticulidae } \\
\text { Stigmella sp. (6) }\end{array}$ & ----------- \\
\hline \multirow[t]{2}{*}{ Conyza bonariensis (buva, voadeira) } & $\begin{array}{l}\text { Gracillariidae } \\
\text { Phyllocnistis sp. (16) } \\
\text { Lithocolletinae gen. nov. }{ }^{(6)}\end{array}$ & $\begin{array}{l}\text { Eulophidae- C. coffeellae (2) } \\
\text { Braconidae- Dolichogenidea sp. (1) } \\
\text {---------- }\end{array}$ \\
\hline & Morfoespécie Não Identificada (2) & Eulophidae- C. coffeellae (2) \\
\hline \multirow[t]{2}{*}{ Ipomoea cairica (corda-de-viola) } & $\begin{array}{l}\text { Acrolepiidae } \\
\text { A. xylophragma (2) }\end{array}$ & Braconidae- Dolichogenidea sp. (2) \\
\hline & $\begin{array}{l}\text { Gracillariidae } \\
\text { Lithocolletinae gen. nov. (1) }\end{array}$ & ------- \\
\hline $\begin{array}{l}\text { Fabaceae } \\
\text { Desmodium incanum (pega-pega) } \\
\text { Malvaceae }\end{array}$ & $\begin{array}{l}\text { Gracillariidae } \\
\text { Porphyrosela sp. }(2) \\
\text { Tischeriidae }\end{array}$ & ------ \\
\hline Sida urens (guanxuma, guaxima) & $\begin{array}{l}\text { Tischeria sp. (6) } \\
\text { Gracillariidae } \\
\text { Phyllocnistis sp. (1) }\end{array}$ & Braconidae- Bracon sp. 1 (1) \\
\hline $\begin{array}{l}\text { Maranthaceae } \\
\text { Marantha arundinacea (araruta) }\end{array}$ & $\begin{array}{l}\text { Gelechiidae } \\
\text { Morfoespécie } 3^{(4)}(36)\end{array}$ & ------------ \\
\hline Portulacaceae & Gelechiidae & \\
\hline Talinum paniculatum (maria-gorda) & Morfoespécie 1 (9) & Braconidae-C. coffeellae (13); Pholetesor sp. 1(5) \\
\hline Rubiaceae & Gelechiidae & \\
\hline Richardia brasiliensis (poaia-branca) & Morfoespécie $2^{(4)}(10)$ & Eulophidae- Chrysocharis sp. 2 (1) \\
\hline Solanaceae & Acrolepiidae & \\
\hline Solanum laxum (joá-cipó) & A. xylophragma (2) & Braconidae- Dolichogenidea sp. (1) \\
\hline Solanum americanum (erva-moura) & A. xylophragma (42) & $\begin{array}{l}\text { Braconidae-Dolichogenidea sp. (6); Bracon sp. } \\
1 \text { (3); Bracon sp. } 2^{(1)}\end{array}$ \\
\hline Solanum commersonii (batata-silvestre) & A. xylophragma ${ }^{(6)}$ & ----------- \\
\hline
\end{tabular}


Tabela 1 - Continuação

\begin{tabular}{|c|c|c|}
\hline $\begin{array}{l}\text { Plantas hospedeiras } \\
\text { família-espécie } \\
\text { (Nome comum) }\end{array}$ & $\begin{array}{l}\text { Lepidópteros minadores } \\
\text { família-espécie } \\
\text { (No de emergidos) }\end{array}$ & $\begin{array}{l}\text { Parasitoides família-espécie } \\
\text { ( } \mathrm{N}^{o} \text { de emergidos) }\end{array}$ \\
\hline $\begin{array}{l}\text { Solanum mauritianum(cuvitinga, } \\
\text { fumo-bravo) }\end{array}$ & A. $x$ ylophragma ${ }^{(6)}$ & ----------- \\
\hline $\begin{array}{l}\text { Solanum pseudocapsicum } \\
\text { (laranjinha-do-mato) }\end{array}$ & A. xylophragma ${ }^{(6)}$ & - \\
\hline Nicotiana alata (fumo-de-jardim) & A. xylophragma ${ }^{(6)}$ & ---------- \\
\hline Onagraceae & Gracillariidae & \\
\hline Ludwigia elegans & Phyllocnistis sp. ${ }^{(6)}$ & \\
\hline
\end{tabular}

${ }^{1}$ Espécie nova;

${ }^{2}$ Provavelmente espécie nova;

${ }^{3}$ Gênero novo;

${ }^{4}$ Provavelmente gênero novo;

${ }^{5}$ Provavelmente família nova;

${ }^{6}$ Não emergiram

\section{DISCUSSÃO}

Costa Lima (1945) comentou que a maioria das espécies de Gracillariidae na fase jovem tem hábito minador e ataca folhas ou o pericarpo de frutos. $\mathrm{O}$ primeiro registro de Phyllocnistis sp. no Brasil foi feito por Costa; Pereira (2001) em São Paulo, sendo que a espécie registrada por esses autores é a mesma referida no presente estudo. Esta espécie de Phylloc- nistis é nova e encontra-se em fase de descrição. No Brasil, os registros de espécies de Porphyrosela foram feitos por Silva et al. (1968) e Benavides; Monteiro (1996). Entretanto, até o momento não há estudos sobre espécies de Cameraria no país.

No Brasil, algumas espécies de Gelechiidae são bastante estudadas, por serem importantes pragas em culturas de interesse econômico (Costa LiMA, 1945; GALLO et al., 2002).

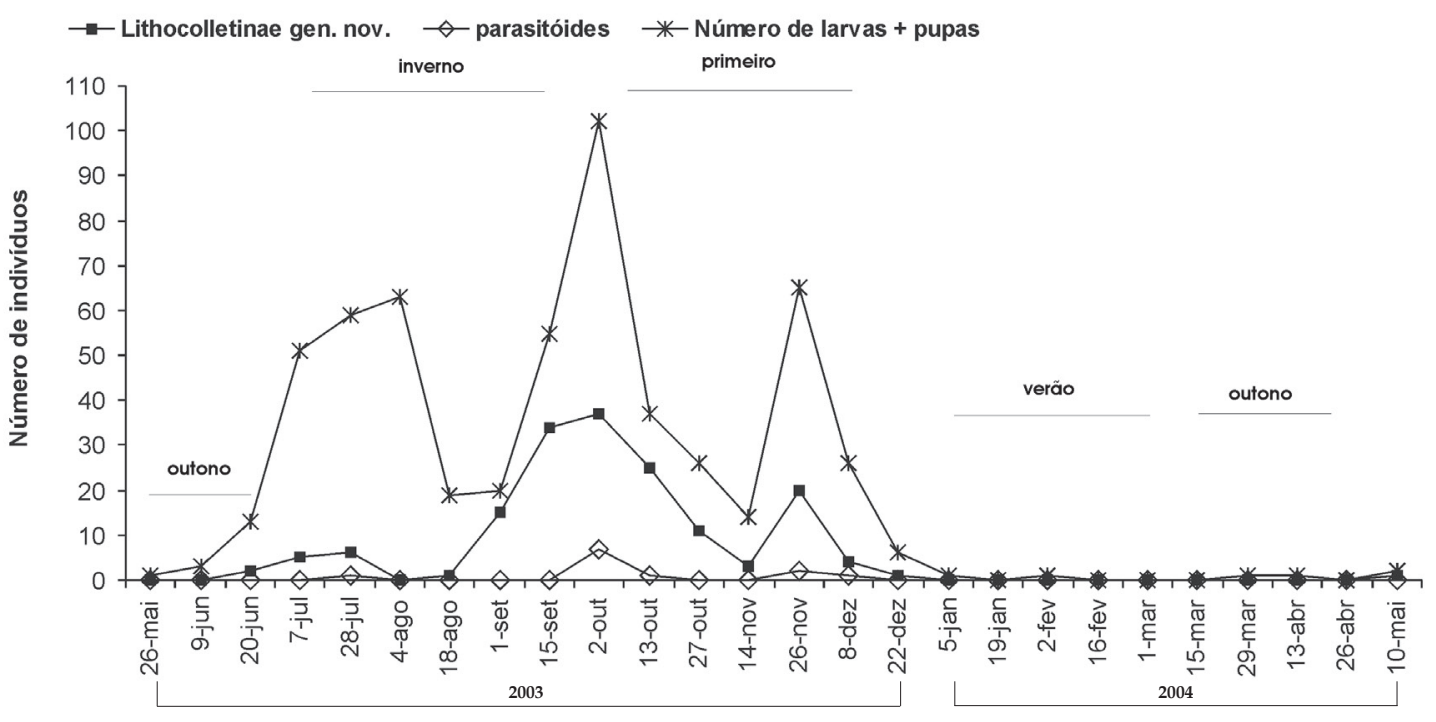

Data das amostragens

Fig. 2- Número de larvas e pupas amostradas em plantas de crescimento espontâneo, em pomar de tangoreiro 'Murcott' e número de adultos de Lithocolletinae gen. nov. e de parasitoides emergidos em laboratório. Montenegro, RS (maio/2003 a maio/2004). 


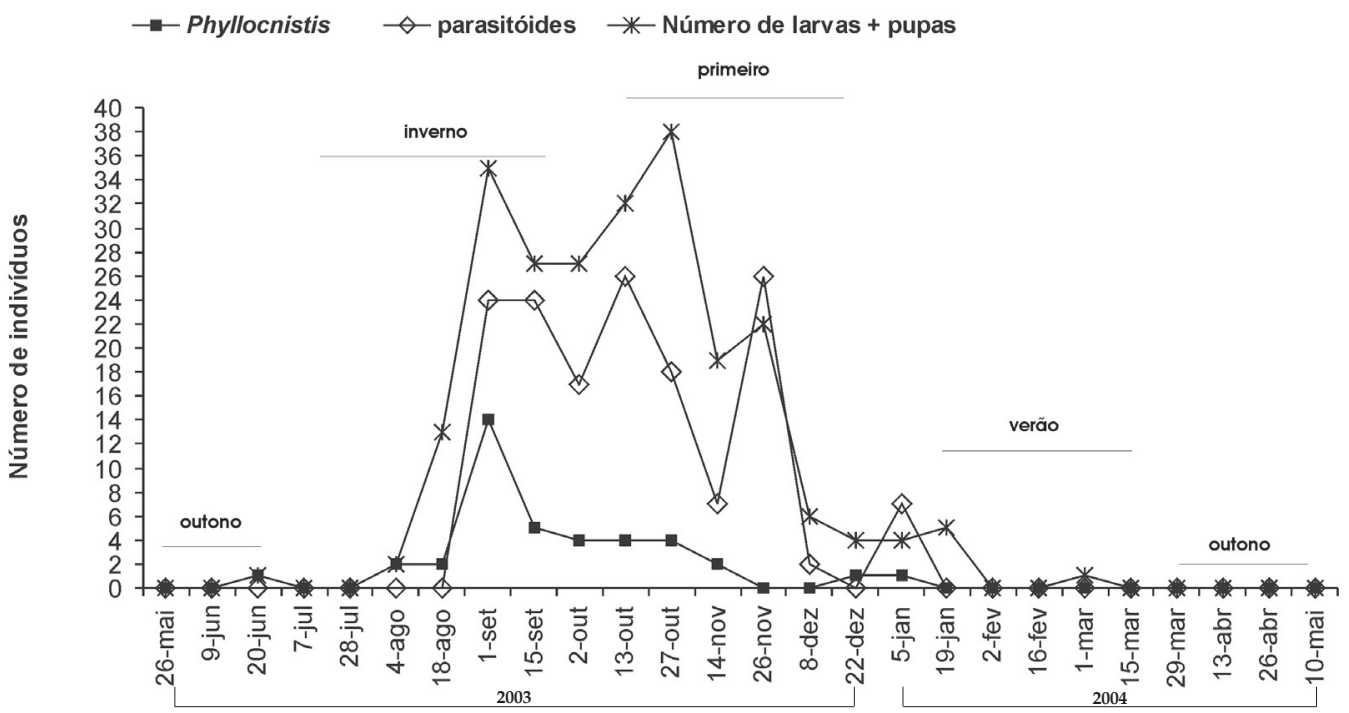

Data das amostragens

Fig. 3- Número de larvas e pupas amostradas em plantas de crescimento espontâneo, em pomar de tangor 'Murcott' e número de adultos de Phyllocnistis sp. e de parasitoides emergidos em laboratório. Montenegro, RS (maio/2003 a maio/2004).

A. xylophragma é referido no Brasil como minador de Solanum mauritianum Scop., 1788 (Solanaceae) (Pedrosa-Macedoet al., 2003). Costa Lima (1945) refere os representantes de Nepticulidae como minadores de folhas e pericarpo de frutos, e os de Tischeriidae como minadores de folhas. No Brasil, a maioria dos representantes de Tischeriidae pertence ao gênero Tischeria. Silva et al. (1968) relataram Tischeria sp. em folhas de malváceas nativas.

Quanto à variação sazonal dos lepidópteros minadores e seus parasitoides, verificou-se que Lithocolletinae gen. nov. ocorreu o ano todo, entretanto a maior frequência desse foi registrada no inverno ena primavera (Fig. 2). No verão e no segundo outono, coletaram-se apenas seis larvas e duas pupas e não se obteve a emergência em laboratório. Apesar de haver poucos indivíduos parasitoides associados à Lithocolletinae gen. nov., estes tiveram uma sincronia de ocorrência com o minador. Imaturos de Lithocolletinae gen. nov. foram encontrados em Conyza bonariensis (L.) Cronquist, 1943 (Asteraceae), Ipomoea cairica (L.) Sweet, 1827 (Convolvulaceae), e Baccharis anomala DC, 1836 (Asteraceae), sendo esta última à hospedeira preferencial deste minador, a qual foi coletada ao longo de todo o período de estudo. Assim, o registro de poucos indivíduos no verão e no outono e a não emergência de adultos das plantas amostradas nesse período, pode indicar que a ocorrência deste minador foi dependente não somente da presença do seu hospedeiro, mas também das condições climáticas.

Phyllocnistissp. teveexpressivaocorrêncianoinverno e na primavera, porém, no outono e no verão, as coletas foraminexpressivas(Fig.3). Osparasitoidesassociadosa esseminador tambémocorreramsimultaneamentecom o minador. Emalgumas ocasiões onúmero de parasitoidessuperouonúmerodelarvasepupasamostradas. Este fato pode ser explicado pela ocorrência de parasitoide gregário C. coffeellae. Como o hospedeiro preferencial de Phyllocnistissp. foi B. anomala, a qualocorreoanointeiro,os resultadossugeremumapossívelpreferênciadesteminador pelos períodos de temperaturas mais amenas. Além deste hospedeiro, imaturos desteminador foram amostradosem C. bonariensis, Sida urens L., 1759 (Malvaceae) e Ludwigia elegans (Cambess.) H. Hara, 1953 (Onagraceae). A única espécie de minador para a qual existem estudos no Rio Grande do Sul é $P$. citrella, para esta espécie há uma relação entre seu tamanho populacional e a presença de folhas novas de citros e também de condições meteorológicas favoráveis, caracterizada pela ausência de ataque na primeira brotação da cultura, iniciada no final do inverno (Greve; Redaelli, 2006). Como Phyllocnistis sp. tem uma boa ocorrência no inverno e é atacada por algumas espécies de parasitoides em comum com $P$. citrella, torna-se um importante reservatório de inimigos naturais desta praga. Nesse contexto, B. anomala é muito importante como hospedeiro alternativo de Phyllocnistis sp. 
Larvas e pupas de A. xylophragma foram amostradas de forma expressiva no inverno e primavera e com menor frequência no verão. Durante o outono não se amostraram imaturos deste minador. Os parasitoides de A. xylophragma tiveram uma ocorrência simultânea (Fig. 4). Imaturos deste minador foram amostrados em várias espécies de solanáceas, tais como Solanum laxum Spreng., 1825, S. americanum Mill, 1768, S. commersonii Dunal, 1814, S. mauritianum Scop., 1788, S. pseudocapsicum L., 1753 e Nicotiana alata Link \& Otto, 1828. A ocorrência deste minador está bastante associada às condições climáticas, tendo em vista que no verão foram amostrados poucos imaturos e no outono estes não foram coletados, muito embora as plantas hospedeiras preferenciais estivessem sempre presentes no local, por vegetarem o ano todo.

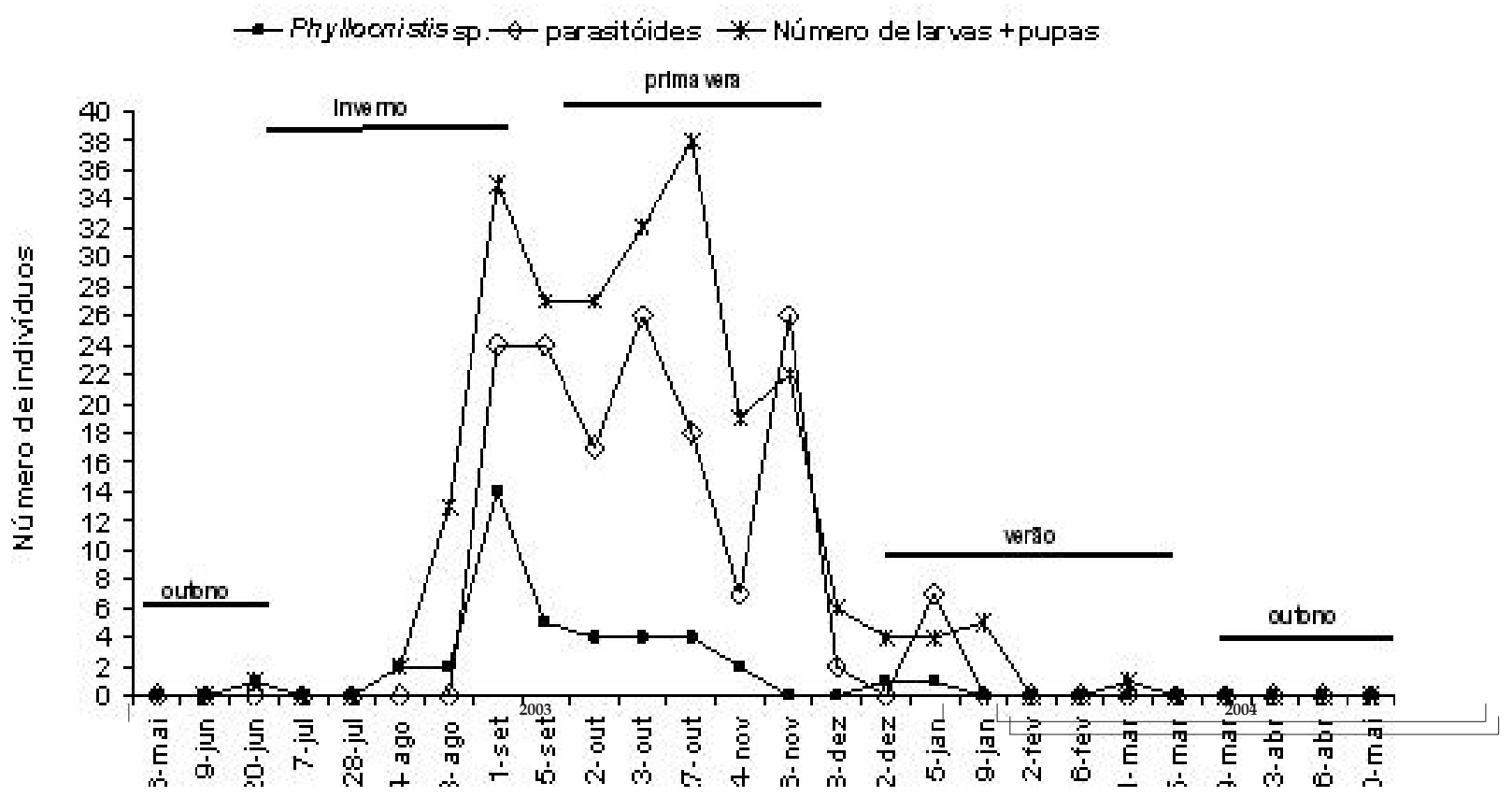

Fig. 4- Número de larvas e pupas amostradas em plantas de crescimento esponeo, em pomar de tangor 'Murcott' e número de adultos de Antispastis xylophragma e de parasitoides emergidos em laboratório. Montenegro, RS (maio/2003 a maio/2004).

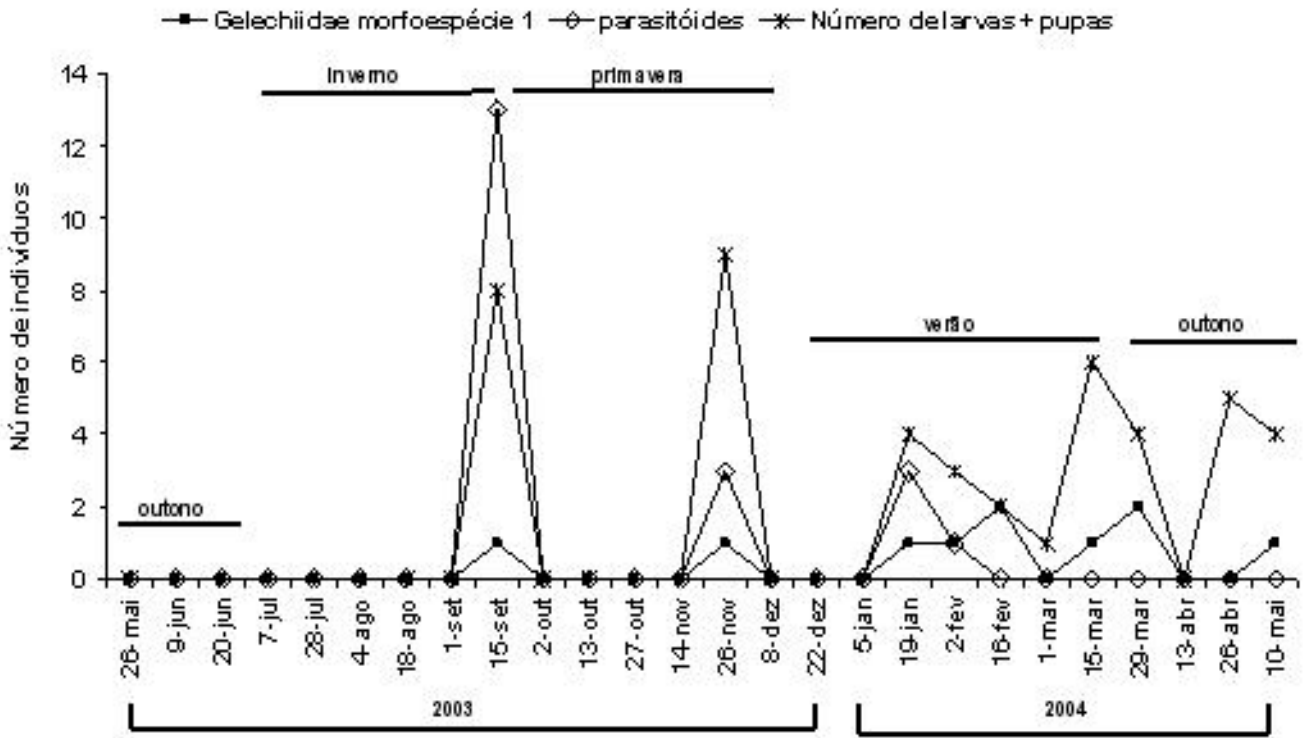

Datadasamotragens

Fig. 5- Número de larvas e pupas amostradas em plantas de crescimento espontâneo, em pomar de tangor 'Murcott' e número de adultos de Gelechiidae morfoespécie 1 e de parasitoides emergidos em laboratório. Montenegro, RS (maio/2003 a maio/2004). 
Imaturos da morfoespécie 1 deGelechiidaeforam coletados praticamente durante todo o período de estudo, porém sem evidenciar qualquer preferência por um período específico e com ampla variação de frequência nas diferentes estações (Fig. 5). Observou-se que os picos de larvas, adultos e de parasitoides foram bastante sincronizados. Constatou-se em uma ocasião, um número grande de parasitoides emergidos, maior que o de larvas coletadas, devido ao parasitismo de $C$. coffeellae. Os imaturos deste minador foram amostrados em $B$. anomala e Talinum paniculatum (Jacq.) Gaertn., 1791 (Portulacaceae), sendo esta última a hospedeira preferencial. De acordo com KissmanN; Groth (2000) T. paniculatum é uma planta anual de ciclo curto. Isso parece indicar que este minador é mais dependente da presença de seu hospedeiro do que das condições climáticas, o que explicaria os diversos picos de ocorrência ao longo de todo o período.

Quanto aos fatores meteorológicos, constatou-se uma precipitação pluviométrica média de $0,8 \mathrm{~mm} /$ dia (Fig. 6). Comparando-se este valor médio da precipitação com os de verões de anos anteriores como 2000/2001 (5,6 mm/dia), 2001/2002 (5,6 mm/dia) e $2002 / 2003$ (3,5 mm/dia), verficou-se que no período de estudo a precipitação foi baixa o que pode ter contribuído para uma menor turgescência das folhas de algumas espécies de plantas, afetando o desenvolvimento normal de alguns minadores. Entretanto, não se registrou correlação entre o número de larvas e pupas amostrado e a precipitação média $(\mathrm{r}=0,2824$; $p=0,1713$ ). Já em relação à temperatura média (Fig. 6) e o número de larvas e pupas amostrado por ocasião, obteve-se uma correlação negativa significativa $(\mathrm{r}=$ $-0,4167 ; p=0,0341$ ), embora baixa (Fig. 7A). Quanto à umidade relativa média, o mesmo foi observado, correlação negativa significativa $(r=-0,7215 ; p<0,0001)$ (Fig.7B). Estes resultadosindicam que os lepidópteros minadores são mais abundantes nos períodos do ano em que as temperaturas são mais baixas e a umidade relativa do ar mais elevada.

Larvas do minador referido neste estudo como morfoespécie não identificada foram amostradas em pequeno número, somente no início do inverno e na primavera, em B. anomala e em Conyza bonariensis. Apenas na primavera foi encontrado C. coffeellae como parasitoide associado.

Larvas e pupas de Tischeria sp. foram amostradas no verão e no outono e, em apenas uma ocasião, emergiu um indivíduo de parasitoide. Coletaram-se os imaturos de Tischeria sp. apenas em S. urens, a qual ocorreu durante todo o período de amostragem. Isso pode sugerir que a ocorrência deste minador está mais associada com as condições climáticas do que com a planta hospedeira.

Larvas de Porphyrosela sp. foram amostradas no verão e no outono, entretanto, a emergência de adultos foi obtida apenas no outono, em uma ocasião. Não foram registrados parasitoides associados a este minador. Porphyrosela sp. foi amostrado na fabácea perene, Desmodium incanum (Sw.) DC., 1825, o que permite supor a dependência deste às condições climáticas.

Outros lepidópteros minadores foram registrados em apenas uma ocasião de amostragem: Cameraria sp. em B. anomola; Gelechiidae morfoespécie 2 em Richardia brasiliensis Gómez (Rubiaceae); e Gelechiidae morfoespécie 3 em Marantha arundinacea L., (Maranthaceae).

Neste trabalho ficou evidenciada a existência de uma considerável diversidade de espécies de insetos minadores e de parasitoides associados a eles. Dentre estas espécies, algumas são registros novos e outras ainda aguardamidentificação, possivelmente também são novas espécies.

A diversidade de organismos, neste habitat, demonstra a importância da manutenção das plantas espontâneas nos agroecossistemas como refúgio e reservatório de agentes de controle biológico, uma vez que nenhum desses minadores estão associados a espécies de citros, apenas $P$. citrella ocorre nessas plantas. O conhecimento, embora incipiente, dos hospedeiros preferenciais dos minadores, da sua época de ocorrência e dos parasitoides associados, permite programar práticas de manejo da vegetação de crescimento espontâneo.

Apesar de $P$. citrella não ter sido observada nas plantas espontâneas presentes no pomar estudado, algumas espécies de parasitoides de outros minadores já foram relatadas em várias regiões do mundo parasitando $P$. citrella, tal como as incluídas nos gêneros Chrysocharis (Schauff et al., 1998), Closterocerus (Browning; PeÑa, 1995; PeÑa et al., 1996), Sympiesis (PeÑa et al., 1996), e Bracon (BARoga, 1968 apud HEPPNER, 1993).

Com base nos resultados obtidos registrou-se 11 espécies de lepidópteros, pertencentes às famílias Gracillariidae, Gelechiidae, Acrolepiidae,Nepticulidae, Tischeriidaee,12espécies dehimenópteros parasitoides das famílias Eulophidae e Braconidae. A ocorrência das larvas e pupas de minadores esteve associada negativamente com os valores de temperatura média e a umidade relativa do ar.

\section{CONCLUSÕES}

1- Phyllocnistis citrella não foi observada nas plantas espontâneas presentes no pomar;

2- Algumas espécies registradas neste estudo já haviam sidorelatadas mundialmentecomo parasitoides de P. citrella. Portanto podem estar atuando como agentes de controle biológico deste inseto na área de estudo. 

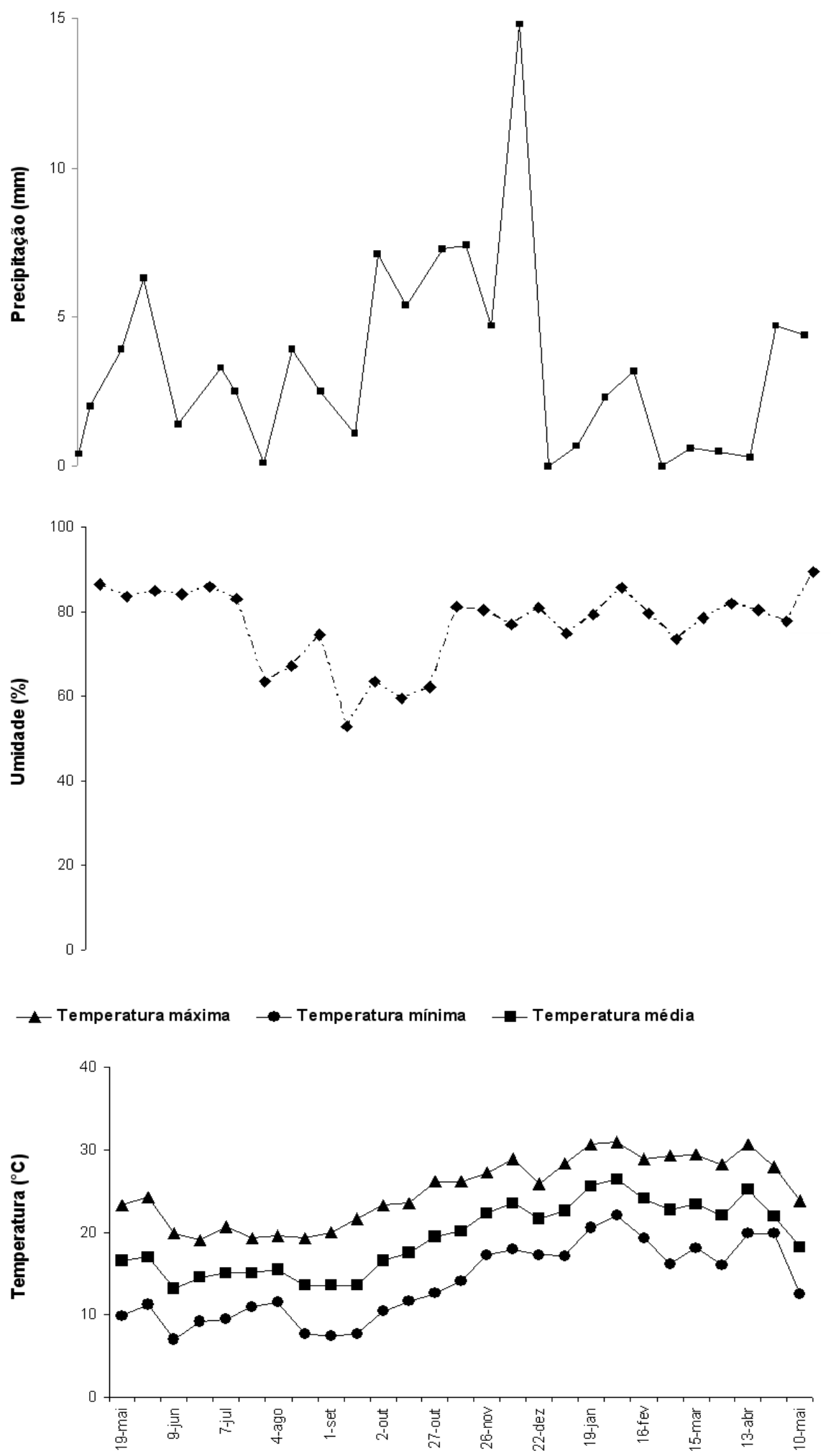

\section{Ocasiões de amostragem}

Fig. 6 - Média acumulada dos valores de umidade relativa do ar, precipitação pluviométrica e temperatura máxima, média e mínima, registrados na quinzena anterior a cada ocasião de amostragem em plantas de crescimento espontâneo, em pomar de tangor 'Murcott', no Município de Montenegro, RS, de maio de 2003 a maio de 2004, Taquari, RS. 

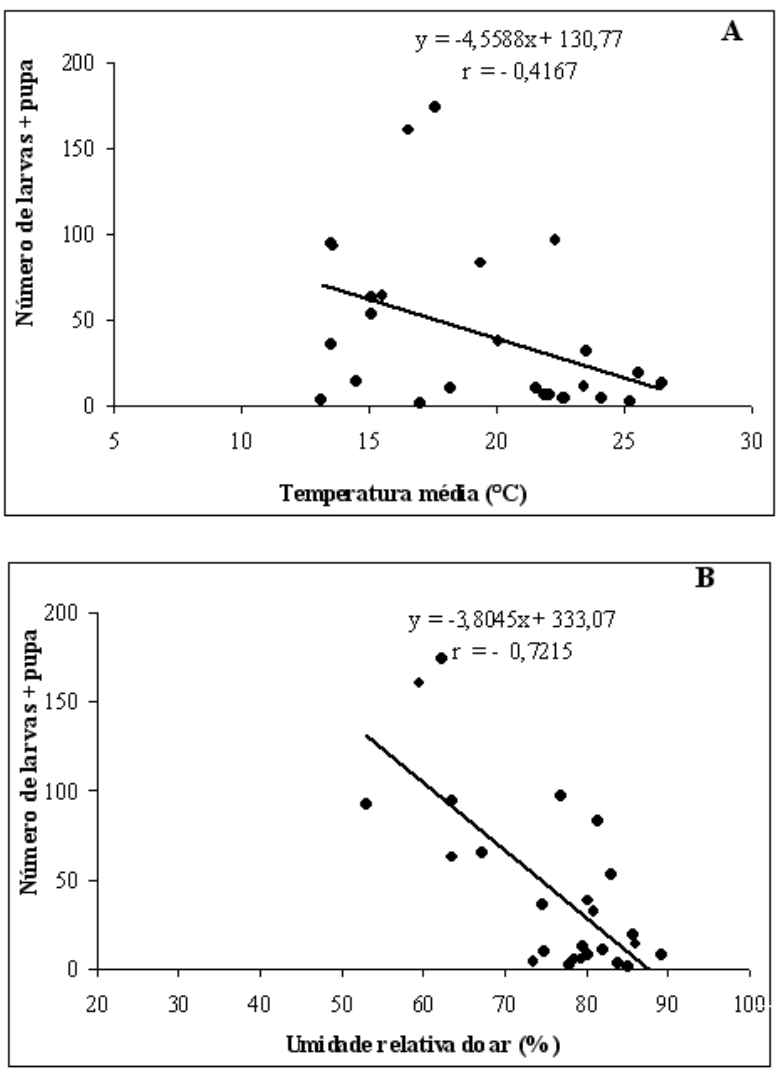

Fig. 7 - Número de larvas e pupas de lepidópteros minadores em função da temperatura média (A) e da umidade relativa do ar (B), por ocasião de amostragem. Montenegro, RS (maio/2003 a maio/2004).

\section{REFERÊNCIAS}

AYRES, M.; AYRES JÚNIOR, M.; AYRES, D.L.; SANTOS, A.S. dos BioEstat 2.0: aplicações estatísticas nas áreas das ciências biológicas e médicas. Belém: Sociedade Civil Mamirauá; Brasília: CNPq, 2000. 272p.

BEGON, M.; MORTIMER, M. Population ecology: an unified study of animals and plants. Oxford: Blackwell Scientific Publications, 1986. 219p.

BEGON, M.; HARPER, J.L.; TOWNSEND, C.R. Ecology: individuals, populations and communities. Oxford: Blackwell Science, 1990. 1068p.

BENAVIDES, M.T.; MONTEIRO, R.F. Ecologia e padrão de alimentação do minador, Porphyrosela sp. (Lep.: Gracilariidae) (sic) em Centrosema pubescens Benth (Leg.: Papilionoideae). In: CONGRESSO DE ECOLOGIA DO BRASIL, 3., 1996, Brasília, DF. Resumos. Brasília, 1996. p.347.

BROWNING, H.W.; PEÑA, J.E. Biological control of the citrus leafminer by its native parasitoids and predators. Citrus Industry, v.76, p.46-48, 1995.

BYERS, J.A. Leaf-mining insects, 2002. Disponível em: <http://www. wcrl.ars.usda.gov/cec/insects/leafmine.htm> Acesso em: 27 dez. 2004.
CARLETTI, E. Insectos de Argentina y el mundo, 2004. Disponível em: <http:/ / www.axxon.com.ar/mus/ info/art-DondeHallarInsectos.htm $>$ Acesso em: 27 dez. 2004.

COSTA, V.A.; PEREIRA, C.F. Ocorrência de Phyllocnistis sp. (Lep.: Gracillariidae) e seus parasitóides (Hym.: Chalcidoidea) em buva (Conyza bonariensis). In: SIMPÓSIO DE CONTROLE BIOLÓGICO, 7., 2001, Poços de Caldas, MG. Resumos. Poços de Caldas, 2001. p.322.

COSTA LIMA, A. Insetos do Brasil: Rio de Janeiro: Escola Nacional de Agronomia, 1945. Tomo 5, $1^{\text {a }}$ parte, Lepidópteros. 379p.

GALLO, D.; NAKANO, O.; SILVEIRA NETO, S.; CARVALHO, R.P.L.; BAPTISTA, G.C. DE; BERTI FILHO, E.; PARRA, J.R.P.; ZUCCHI, R.A.; ALVES, S.B.; VENDRAMIM, J.D.; MARCHINI, L.C.; LOPES, J.R.S.; OMOTO, C. Entomologia agrícola. Piracicaba: Fundação de Estudos Agrários "Luiz de Queiroz", 2002. 920p.

GAULD, I.D.; HANSON, P.E. The evolution, classification and identification of the Hymenoptera. In: HANSON, P.E.; GAULD, I.D. (Ed.). The Hymenoptera of Costa Rica. Oxford: Oxford University Press, 1995. p.138-156. GREVE, C; REDAELLI, L.R. Variação sazonal dos estágios imaturos de Phyllocnistis citrella Stainton (Lepidop- 
tera: Gracillariidae) em pomares de Citrus sinensis sob dois sistemas de cultivo. Neotropical Entomology, v.35, n.6, p.828-833, 2006.

HEPPNER, J.B. Citrus leafminer, Phyllocnistis citrella, in Florida (Lepidoptera: Gracillariidae: Phyllocnistinae). Tropical Lepidoptera, v.4, n.1, p.49-64, 1993.

HESPENHEIDE, H.A. Bionomics of leaf-mining insects. Annual Review of Entomology, v.36, p.535-560, 1991.

JAHNKE, S.M.; REDAELLI, L.R.; DIEFENBACH, L.M.G. Complexo de parasitoides de Phyllocnistis citrella (Lepidoptera, Gracillariidae) em dois pomares de citros em Montenegro, RS, Brasil. Iheringia. Série Zoologia, v.95, n.4, p.359-363, 2005

KISSMANN, K.G.; GROTH, D. Plantas infestantes e nocivas. 2.ed. São Paulo: BASF Brasileira, Indústrias Químicas, 2000. v.3, 726p.

KNAPP, J.L.; ALBRIGO, L.G.; BROWNING, H.W.; BULLOCK, R.C.; HEPPNER, J.B.; ALL, D.G.; HOY, M.A.; NGUYEN, R.; PEÑA, J.E.; STANSLY, P.A. Citrus leafminer, Phyllocnistis citrella Stainton: current status in Florida. Gainesville: Florida Cooperative Extension Service Institute of Food and Agricultural Sciences, University of Florida, 1995. 34p.

PEDROSA-MACEDO, J.H.; OLCKERS, T.; VITORINO, M.D.; CAXAMBU, M.G. Phytophagous arthropods associated with Solanum mauritianum Scopoli (Solanaceae) in the first Plateau of Paraná, Brazil: a cooperative project on biological control of weeds between Brazil and South America. Neotropical Entomology, v.32, n.3, p.519-522, 2003.

PEÑA, J.E; DUNCAN, R.; BROWNING, H.W. Seasonal abundance of Phyllocnistis citrella (Lepidoptera: Gracillariidae) and its parasitoids in South Florida citrus. Enviromental Entomology, v.25, n.3, p.698-702, 1996.
RODRIGUEZ, O.; VIÉGAS, F.; POMPEU JÚNIOR, J.; AMARO, A.A. (Coord.). Citricultura brasileira. Campinas: Fundação Cargill, 1991. 492p.

SCHAFFER, B.; PEÑA, J.E.; COLLS, A.M.; HUNSBERGER, A. Citrus leafminer (Lepidoptera: Gracillariidae) in lime: assessment of leaf damage and effects on photosynthesis. Crop Protection, v.16, n.4, p.337-343, 1997.

SHAUFF, M.E.; LASALLE, J.; COOTE, L.D. Subfamily Entedontinae. In: WHARTON, R.A.; MARSH, P.M.; SHARKEY, M.J. (Ed.). Manual of the new world genera of the family Braconidae (Hymenoptera). Washington, DC: The International Society of Hymenopteristis, 1997. (Special Publication, n.1). p.327-429.

SILVA, A.G.D'ARAÚJO E; GONÇALVES, C.R.; GALVÃO, D.M.; GONÇALVES, A.J.L.; GOMES, J.; SILVA, M. DO. N.; SIMONI, L. de. Quarto catálogo dos insetos que vivem nas plantas do Brasil: seus parasitos e predadores. Rio de Janeiro: Serviço de Defesa Sanitária, Ministério da Agricultura, 1968. Parte 2.Tomo 1. Insetos, hospedeiros e inimigos naturais. 622p.

SOUTHWOOD, T.R.E. Ecological methods: with particular reference to the study of insect populations. London: Chapman and Hall, 1978. 524p.

VERDÚ, M.J. Chalcidoidea (Hymenoptera), parásitos del minador de los cítricos Phyllocnistis citrella (S) (Lep.; Gracillariidae) en España. Levante Agrícola, v.336, p.227230, 1996.

Recebido em 13/3/07

Aceito em 22/6/09 